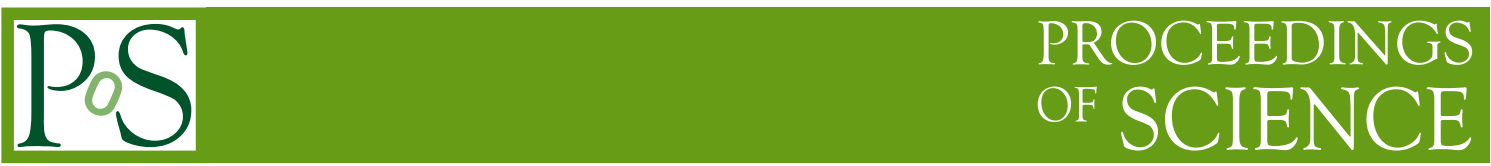

\title{
Recent results from Borexino
}

\section{D'Angelo*}

Dipartimento di Fisica, Università degli Studi e INFN, 20133 Milano, Italy

E-mail: davide.dangelo@mi.infn.it

M. Agostini, K. Altenmüller, S. Appel, V. Atroshchenko, G. Bellini, J. Benziger, D. Bick, G. Bonfini, D. Bravo, B. Caccianiga, F. Calaprice, A. Caminata, M. Carlini, P. Cavalcante, A. Chepurnov, K. Choi, S. Davini, A. Derbin, L. Di Noto, I. Drachnev, A. Etenko, K. Fomenko, D. Franco, F. Gabriele, C. Galbiati, C. Ghiano, M. Giammarchi, M. Goeger-Neff, A. Goretti, M. Gromov, C. Hagner, E. Hungerford, Aldo lanni, Andrea lanni, A. Jany, K. Jedrzejczak, D. Jeschke, V. Kobychev, D. Korablev, G. Korga, D. Kryn, M. Laubenstein, B. Lehnert, E. Litvinovich, F. Lombardi, P. Lombardi, L. Ludhova, G. Lukyanchenko, I. Machulin, S. Manecki, W. Maneschg, G. Manuzio, S. Marcocci, E. Meroni, M. Meyer, L. Miramonti, M. Misiaszek, M. Montuschi, P. Mosteiro, V. Muratova, B. Neumair, L. Oberauer, M. Obolensky, F. Ortica, M. Pallavicini, L. Papp, A. Pocar, G. Ranucci, A. Razeto, A. Re, A. Romani, R. Roncin, N. Rossi, S. Schönert, D. Semenov, M. Skorokhvatov, O. Smirnov, A. Sotnikov, S. Sukhotin, Y. Suvorov, R. Tartaglia, G. Testera, J. Thurn, M. Toropova, E. Unzhakov, A. Vishneva, R.B. Vogelaar, F. von Feilitzsch, H. Wang, S. Weinz, J. Winter, M. Wojcik, M. Wurm, Z. Yokley, O. Zaimidoroga, S. Zavatarelli, K. Zuber, and G. Zuzel (Borexino Collaboration)

The Borexino experiment is operational at Laboratori Nazionali del Gran Sasso since 2007. Its technical distinctive feature is the unprecedented ultralow background of the inner scintillating core, which is the basis of the outstanding achievements obtained by the experiment. After recalling the main features of the detector, we review the solar and geoneutrino results accumulated so far, with special emphasis to the most recent and prominent results. Specifically, these are on the one hand the measure of the fundamental pp solar neutrino flux, which is the direct probe of the engine mechanism powering our star, and on the other the detection of the geo-neutrino signal with its geophysical implications for the interior of the Earth. The pp-neutrino milestone measurement puts Borexino in the unique situation of being the only experiment able to do solar neutrino spectroscopy over the entire solar spectrum; the counterpart of this peculiar status in the oscillation interpretation of the data is the capability of Borexino alone to perform the full validation across the solar energy range of the MSW-LMA paradigm. We conclude highlighting the plans to fully complete the solar spectroscopy with the missing piece of the CNO neutrinos. If successful, such a measurement would represent the final crowning of the long quest of Borexino to unravel all the properties of the neutrinos from the Sun.

38th International Conference on High Energy Physics

3-10 August 2016

Chicago, USA

${ }^{*}$ Speaker. 


\section{Why solar neutrinos with Borexino}

The Sun is an intense source of neutrinos, produced in nuclear reactions of the p-p chain and of the CNO cycle [1]. Measurements of the individual neutrino fluxes is of paramount importance for both particle physics and astrophysics. The solar neutrino spectrum can be seen in figure 1. Up to a few years ago, spectroscopical measurements were performed by water Cherenkov detectors above $\sim 5 \mathrm{MeV}$ and concerned only ${ }^{8} \mathrm{~B}$ neutrinos for less then $1 \%$ of the total flux. The bulk of neutrinos at low energies were detected only with radiochemical experiments, incapable of resolving the individual components. Neutrinos are emitted in the Sun as electron-flavour neutrinos and may oscillate to a different flavour during the trajectory to the Earth. The MSW-LMA mechanism foresees the survival probability for electron neutrinos on Earth after oscillation is taken into account, as shown in figure 2. Borexino was designed to perform spectroscopy at low energy targeting in particular the flux of the ${ }^{7} \mathrm{Be}$ monochromatic line at $862 \mathrm{keV}$. Borexino has largely exceeded the expected performance and broadened the physics program past the original goal.
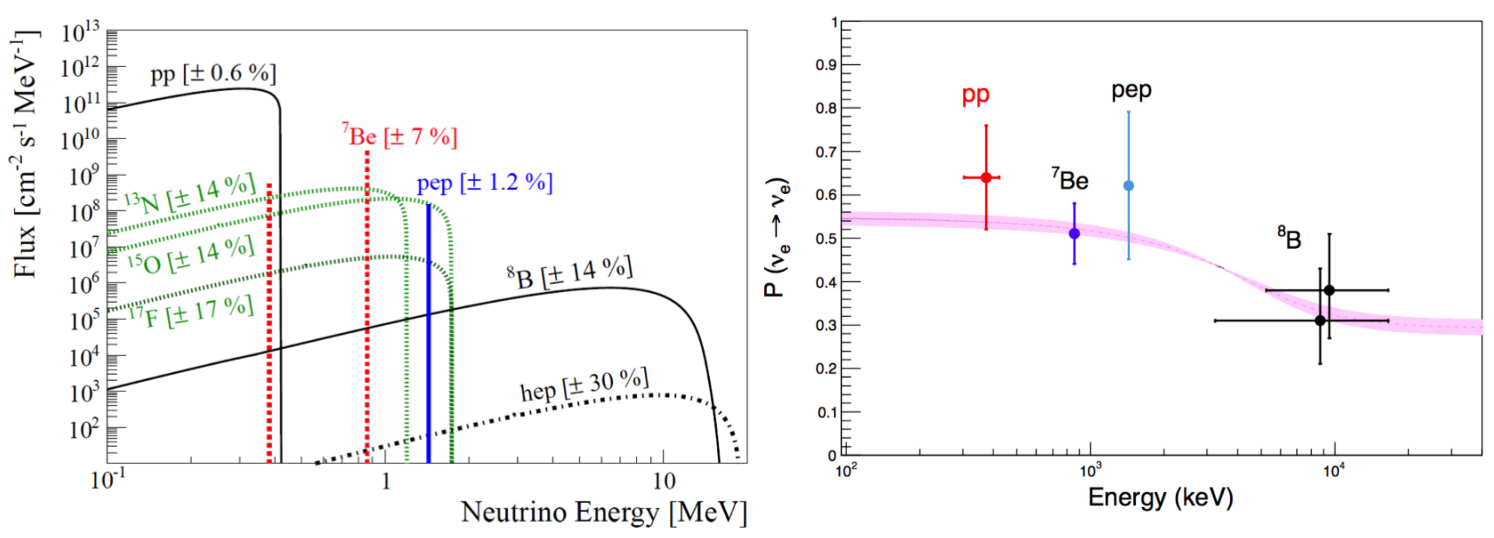

Figure 1: Solar Neutrino Spectrum[1].

Figure 2: Survival probability of electron neutrinos as foreseen by the MSW-LMA model. Data points discussed here are also shown[9].

\section{The Borexino Project}

Detector layout. The Borexino detector [2] is sketched in figure 3. It is located at the Gran Sasso National Laboratories (LNGS) in central Italy, at a depth of 3800 m w.e.. The active mass consists of 278 tons of pseudocumene (PC), doped with $1.5 \mathrm{~g} / 1$ of PPO. The scintillator is contained in a thin $(125 \mu \mathrm{m})$ nylon Inner Vessel (IV), $8.5 \mathrm{~m}$ in diameter. The IV is surrounded by two concentric PC buffers doped with a light quencher. The scintillator and buffers are contained in a Stainless Steel Sphere (SSS) with a diameter of $13.7 \mathrm{~m}$. The SSS is enclosed in a Water Tank (WT), containing 2100 tons of ultra-pure water as an additional shield against backgrounds from the laboratory environment. The scintillation light released upon particle interaction in the pseudocumene is detected by 2212 8" PhotoMultiplier Tubes (PMTs) uniformly distributed on the inner surface of the SSS. Additional 208 8" PMTs instrument the WT and detect the Cherenkov light radiated by cosmic muons that cross the water shield. 
Detector performance. Neutrinos interact via elastic scattering on the electrons of the target material, resulting in $\sim 500$ detected photoelectrons for a $1 \mathrm{MeV}$ recoil electron. The fast time response $(\sim 3 \mathrm{~ns})$ of the scintillating mixture allows to reconstruct the events position by means of a time-offlight technique with $\sim 13 \mathrm{~cm}$ precision for events at the center of the detector. Depending on the analysis, the fiducial volume is defined between 75 tons and 150 tons. The signature of ${ }^{7} \mathrm{Be}$ neutrinos is a Compton-like shoulder at $665 \mathrm{keV}$ in the electron recoil spectrum. The energy resolution $(1 \sigma)$ at ${ }^{7} \mathrm{Be}$ en-

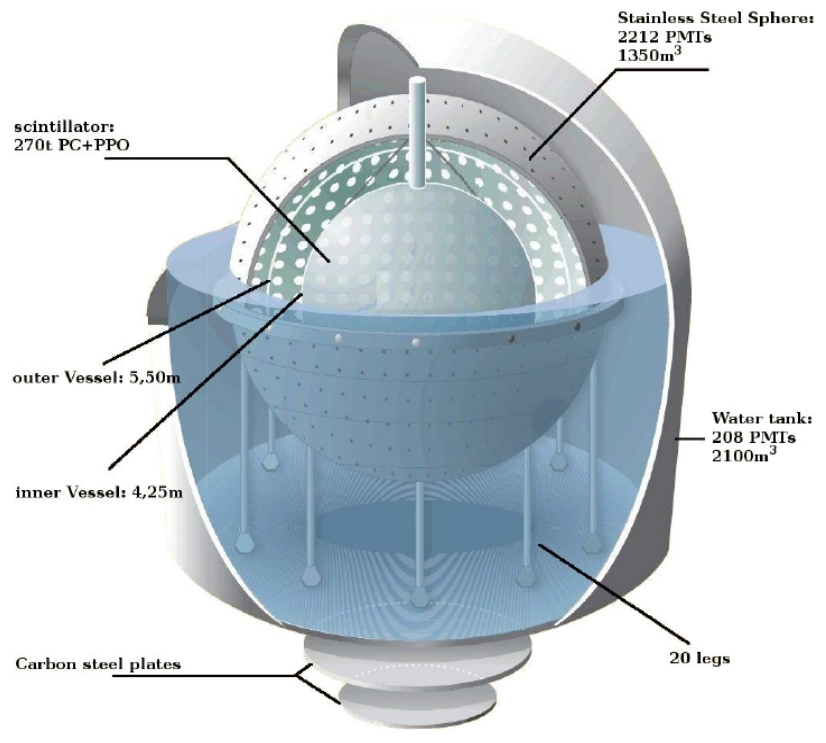

Figure 3: Sketch of the Borexino detector. ergy is as low as $44 \mathrm{keV}$ (or 6.6\%). The inner detector trigger is defined as the coincident firing of at least 20-25 PMTs, corresponding to an energy threshold of $\sim 40-50 \mathrm{keV}$. However the analysis threshold in phase-I was limited by the knowledge of the ${ }^{14} \mathrm{C}$ spectral shape to $\sim 250 \mathrm{keV}$. In phase-II we have successfully reduced our threshold to $165 \mathrm{keV}$ (see Sect. 4). Pulse Shape Analysis (PSA) is performed to identify various classes of events, among which electronic noise, pile-up events, muons, $\alpha$ and $\beta$ particles.

Borexino timeline. The phase-I of Borexino occurred between May 2007 and May 2010. Next, we proceeded to the purification of the scintillator by water extraction and sparging with a specially developed nitrogen gas, low in $\mathrm{Kr}$ and Ar contamination. After looping through the purification plant for six complete cycles, in November 2011 the phase-II of Borexino started and is expected to last at least until the end of 2017. The energy scale uncertainty in the range [0.2$2.0 \mathrm{MeV}$ has been determined at $1.5 \%$ precision, using multiple gamma sources. The position reconstruction algorithm has been tuned using a $\mathrm{Rn}$ source placed in 184 positions inside the active volume. The determination of the fiducial volume is now $-1.3 \%+0.5 \%$. An external calibration campaign has allowed to correctly model the external gamma backgrounds. The calibrations will be repeated before the end of phase-II to demonstrate stability of the detector and to improve the previous results[3].

Experimental backgrounds. The background levels of the Borexino scintillator are summarised in table $1 .{ }^{238} \mathrm{U}$ and ${ }^{232} \mathrm{Th}$ contaminations are unprecedented low and largely exceed the requirements of the experiment. Most other contaminants are at acceptable levels or have been reduced below required limits after purification. We observe the presence of Radon Daughters ${ }^{210} \mathrm{Bi}$ and ${ }^{210} \mathrm{Po}$ out of equilibrium. ${ }^{210} \mathrm{Po}$ is of little concern as it is an $\alpha$ emitter and it can be identified by PSA. The purification campaign has been particularly effective in suppressing ${ }^{85} \mathrm{Kr}$ and ${ }^{210} \mathrm{Bi}$ effectively allowing the measurement of pp neutrino (Sect. 4) and opening the possibility of a measurement of CNO neutrino fluxes (Sect. 7). 


\begin{tabular}{ccc}
\hline Isotope & Before purification & After purification \\
\hline${ }^{14} \mathrm{C} /{ }^{12} \mathrm{C}$ & $(2.69 \pm 0.06) 10^{-18}$ & unchanged \\
${ }^{85} \mathrm{Kr}$ & $(30 \pm 5) \mathrm{cpd} / 100 \mathrm{t}$ & $\leq 5 \mathrm{cpd} / 100 \mathrm{t}$ \\
${ }^{210} \mathrm{Po}$ & $\sim 20 \mathrm{cpd} / \mathrm{t}$ & unchanged \\
${ }^{210} \mathrm{Bi}$ & $\sim 40 \mathrm{cpd} / 100 \mathrm{t}$ & $\sim 20 \mathrm{cpd} / 100 \mathrm{t}$ \\
\hline
\end{tabular}

Table 1: Main background levels in the Borexino scintillator. cpd stands for "counts per day".

\section{Phase-I results}

${ }^{7}$ Be flux. The measurement of the flux of ${ }^{7} \mathrm{Be}$ neutrinos was the primary goal of Borexino. Thanks to the unprecedented background levels, the first observation was published in summer 2007, after only 3 months of data taking. The flux was later reviewed in 2008 and 2011 reducing the error every time. The signal is extracted by a spectral fit along with other neutrino signals and background components. The last measured rate[4] after $741 \mathrm{~d}$ of live time (full phase-I) is $R_{\mathrm{Be}}=46.0 \pm 1.5_{\text {stat }} \pm 1.6_{\text {syst }} / \mathrm{d} / 100 \mathrm{t}$. For the first time the experimental error (4.8\%) is lower the theoretical uncertainty from the Standard Solar Model (7\%). The rate corresponds to a flux of $\Phi_{B e}=(3.10 \pm 0.15) \times 10^{9} \mathrm{~cm}^{-2} \mathrm{~s}^{-1}$ with a survival probability $P_{e e}=0.51 \pm 0.07$ at $862 \mathrm{keV}$.

We have also carefully inspected the data set at the energy of ${ }^{7} \mathrm{Be}$ neutrino scattering looking for an eventual day-night asymmetry[5]. This was foreseen in an alternative MSW scenario, called LOW, also compatible to some extent with the solar neutrino results. Our data is consistent with no asymmetry: $A_{D N}=\Phi_{N}-\Phi_{D} / \Phi=0.001 \pm 0.012_{\text {stat }} \pm 0.007_{\text {syst }}$. With this result, the MSW-LOW mechanism can be ruled out at $8.5 \sigma$ using only solar neutrino data.

Due to the eccentricity of the Earth's orbit, the observed solar neutrino flux is expected to undergo a yearly modulation with amplitude $\pm 3.4 \%$ and a maximum at the beginning of January. The presence of such modulation is the ultimate proof that Borexino is actually observing neutrinos from the Sun. Using a data set of $850 \mathrm{~d}$ (astr. time), we have found $>3 \sigma$ evidence of a modulation at ${ }^{7} \mathrm{Be}$ energy with period $(1.01 \pm 0.07) \mathrm{y}[6]$. Phase, amplitude and average ${ }^{7} \mathrm{Be}$ rate are consistent within $2 \sigma$ with the expectations. An alternative approach uses Lomb-Scargle frequency analysis, confirms this result and doesn't find any other relevant time component in the rate.

${ }^{8} \mathbf{B}$ flux Borexino has measured the ${ }^{8} \mathrm{~B}$ flux down to $3.0 \mathrm{MeV}[7]$. While the much larger water Cherenkov detectors can achieve better precision, Borexino holds the lowest threshold achieved so far. Lowering the threshold on ${ }^{8} \mathrm{~B}$ spectroscopy represents one of the key features to inspect the transition region of the LMA scenario (see Sect. 1). The measured rate is $R_{B}=0.22 \pm 0.04_{\text {stat }} \pm$ $0.01_{\text {syst }}$ and corresponds to a flux of $\Phi_{B}=\left(2.4 \pm 0.4_{\text {stat }} \pm 0.1_{\text {syst }}\right) \times 10^{6} \mathrm{~cm}^{-2} \mathrm{~s}^{-1}$. The flux above $5 \mathrm{MeV}$ is in good consistency with other results.

pep flux and CNO limits. The pep neutrino energy lies at the boundary between the Vacuum and the Transition region of the MSW survival probability. In the same energy region are the so far undetected neutrinos from the $\mathrm{CNO}$ cycle reactions. At this energy the cosmogenic background of ${ }^{11} \mathrm{C}$ is overwhelming the signal by about an order of magnitude. We suppress this background 
exploiting the three-fold coincidence between the parent muon, the ${ }^{11} \mathrm{C}$ and the neutron most often accompanying its production. By excluding the regions of the active volume close to the muon track or to the neutron capture point for a few half lives of ${ }^{11} \mathrm{C}$ ( $\left.29 \mathrm{~min}\right)$, we select about $50 \%$ of the exposure where the ${ }^{11} \mathrm{C}$ background is only $10 \%$ of the total. Another powerful handle to distinguish neutrinos from ${ }^{11} \mathrm{C}$ is PSA as the scintillation due to annihilation gamma-rays accompanying the ${ }^{11} \mathrm{C} \mathrm{e}^{+}$-decay may be delayed when released by ortho-positronium bound state. The rate of pep neutrinos has been extracted with a multivariate analysis based of the energy of the event, the distance from the center of the detector and a pulse shape parameter[6]. The rate is $R_{\text {pep }}=\left(3.1 \pm 0.6_{\text {stat }} \pm 0.3_{\text {syst }}\right) / \mathrm{d} / 100 \mathrm{t}$ corresponding to a flux of $\Phi_{\text {pep }}=(1.6 \pm 0.3) \times 10^{8} \mathrm{~cm}^{-2} \mathrm{~s}^{-1}$ and a survival probability of $P_{\text {pep }}^{e e}=0.62 \pm 0.17$ at $1.44 \mathrm{MeV}$ [8]. The flux of CNO neutrinos could not be extracted due to the spectral shape degeneracy with the ${ }^{210} \mathrm{Bi}$ background. The strongest upper limit available to date has been however obtained from this analysis. The rate of CNO neutrinos is $R<7.1 / \mathrm{d} / 100 \mathrm{t}$ at $95 \%$ C.L. corresponding to $\Phi_{\mathrm{CNO}}<7.7 \times 10^{8} \mathrm{~cm}^{-2} \mathrm{~s}^{-1}$. Improving this analysis using the phase-II data to reduce errors and possibly detect CNO neutrinos is our main goal for 2017 (Sect. 6).

\section{4. pp neutrino flux}

Neutrino from the fundamental pp reaction which powers the Sun are the most elusive to detection as their energy only spans up to $420 \mathrm{keV}$, corresponding to $264 \mathrm{keV}$ in the recoil electron energy spectrum. The expected rate is $(131 \pm 2) / \mathrm{d} / 100 \mathrm{t}$. Though the Borexino scintillator was obtained from selected ancient oil batches to have a low ${ }^{14} \mathrm{C}$ content, the detector's trigger rate of $\sim 30 \mathrm{~s}^{-1} \quad(\sim 50 \mathrm{keV}$ hardware threshold $)$ is largely dominated by ${ }^{14} \mathrm{C}$ decays whose spectrum extends up to $\sim 220 \mathrm{keV}$. We correctly modelled the shape of ${ }^{14} \mathrm{C}$ and constrained its rate studying the events that accidentally fall within the acquisition window opened by another uncorrelated event, effec-

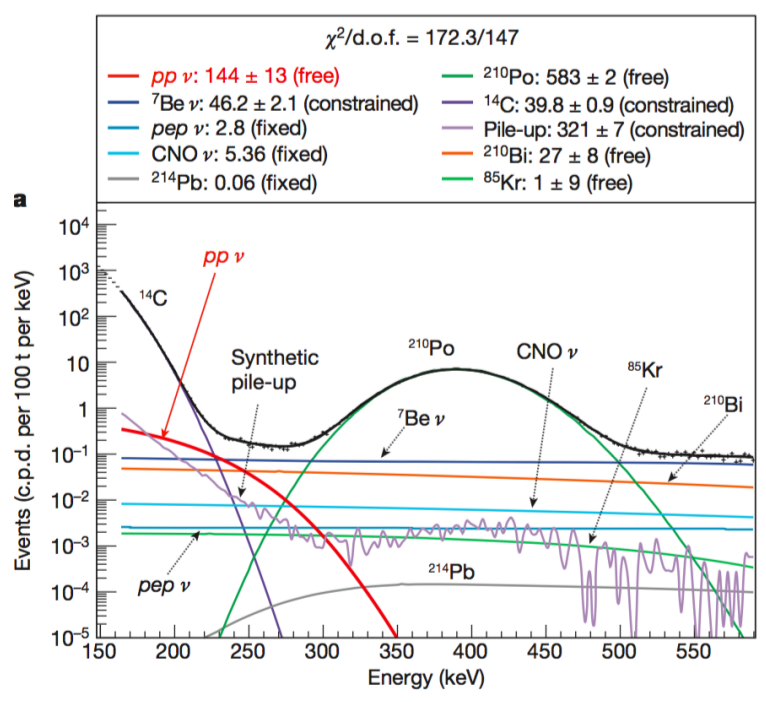

Figure 4: Fit to the energy spectrum for pp neutrino analysis[9]. tively avoiding trigger threshold distortion.

Moreover ${ }^{14} \mathrm{C}$ decays piled-up with another event constitute a spectral shape of their own. We have determined pile-up shape overlapping uncorrelated events with regular data and we added it to the spectral fit with an independently constrained rate. Using data from January 2012 to May 2013 ( 85 ton.yr), we obtained the first direct detection of pp neutrinos. We performed the fit in the $[165,590] \mathrm{keV}$ energy range as shown in figure 4 . The pp neutrino rate was determined to be $144 \pm 13_{\text {stat }} \pm 10_{\text {sys }} / \mathrm{d} / 100 \mathrm{t}$, rejecting the null hypothesis at $10 \sigma$. The result interpretation is threefold: (i) assuming the SSM is correct, our result indicates a survival probability of $(0.64 \pm 0.12)$ 


\begin{tabular}{llll}
\hline Species & Rate $[\mathrm{cpd} / 100 \mathrm{t}]$ & Flux $\left[\mathrm{cm}^{-2} \mathrm{~s}^{-1}\right]$ & Reference \\
\hline $\mathrm{pp}$ & $144 \pm 13 \pm 10$ & $(6.6 \pm 0.7) \times 10^{10}$ & Nature 512 (2014) 7515 \\
${ }^{7} \mathrm{Be}$ & $46.0 \pm 1.5 \pm 1.5$ & $(3.1 \pm 0.15) \times 10^{9}$ & PRL 107 (20122) 141302 \\
pep & $3.1 \pm 0.6 \pm 0.3$ & $(1.6 \pm 0.3) \times 10^{8}$ & PRL 108 (2012) 051302 \\
${ }^{8} \mathrm{~B}$ & $0.22 \pm 0.04 \pm 0.01$ & $(2.4 \pm 0.4) \times 10^{6}$ & PRD 82 (2010) 033006 \\
$\mathrm{CNO}$ & $<7.9(95 \%$ C.L.) & $<7.7(95 \%$ C.L.) & PRL 108 (2012) 051302 \\
\hline
\end{tabular}

Table 2: Summary of Borexino solar neutrino results. cpd stands for "counts per day".

in confirmation of neutrino oscillations with MSW-LMA mechanism in the vacuum dominated region; (ii) assuming the neutrino oscillation model is correct, our result converts to an unoscillated solar neutrino flux of $(6.6 \pm 0.7) \times 10^{10} \mathrm{~cm}^{-2} \mathrm{~s}^{-1}$, confirming the SSM predictions on neutrinos emitted by the reaction most fundamentally bound to the energy production in the Sun; (iii) if both previous assumptions are made, the Borexino result says that the luminosity of the Sun in neutrinos matches the photon luminosity, which is a proof of the Sun stability over a period of $10^{5}$ years. The measurement of the pp neutrino flux by Borexino was published by the Nature Journal[9].

Survival probability after Borexino. Table 2 shows a summary of the Borexino low energy neutrino spectroscopy discussed here. These are also reported in figure 2, already introduced in Sect. 1, superimposed to the survival probability of solar electron neutrinos. The confirmation of the MSW-LMA model over a wide range of energies is striking. However, the unexplored transition region $(1-3 \mathrm{MeV})$ still has room for alternative models and new physics. There are two ways to test these hypotheses or confirm MSW-LMA: (i) reducing the errors on pep and ${ }^{7} \mathrm{Be}$ neutrino fluxes and (ii) lowering the threshold on ${ }^{8} \mathrm{~B}$ neutrinos to observe (or not) the expected upturn of the spectrum. The analyses we are currently performing on Borexino Phase-II data follow both approaches.

\section{Geo-neutrinos}

Geo-neutrinos are anti-neutrinos produced by elements in the radioactive chains of ${ }^{238} \mathrm{U},{ }^{232} \mathrm{Th}$ and by the decay of ${ }^{40} \mathrm{~K}$, with a flux of the order of $\sim 10^{6} \mathrm{~cm}^{-2} \mathrm{~s}^{-1}$. These long lived elements are found in the Earth crust and mantle with unknown abundances. Measuring geo-neutrinos flux can help the development of Earth models and shed light on the heat production mechanism of the planet. Only geo-neutrinos from ${ }^{238} \mathrm{U}$ and ${ }^{232} \mathrm{Th}$ chain elements can be measured by neutrino detectors, via Inverse Beta Decay with a threshold of $1.8 \mathrm{MeV}$. The ratio of $\mathrm{Th} / \mathrm{U}$ is supposed to be $\sim 3.9$ from the anal-

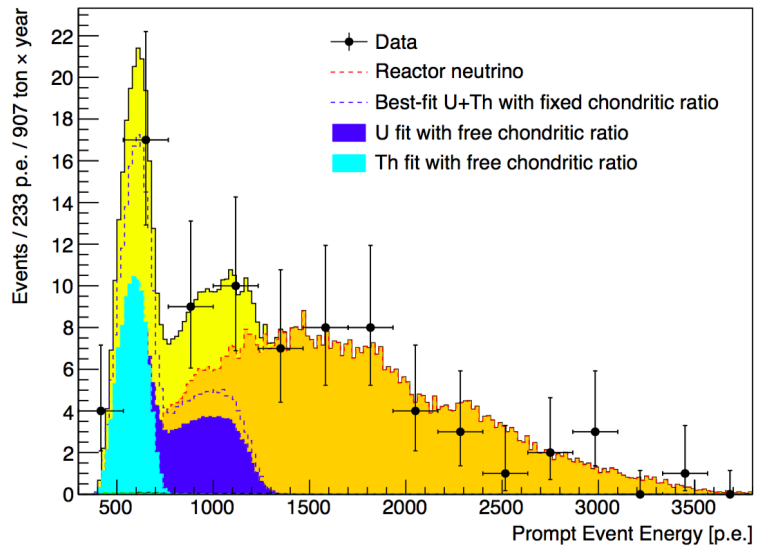

Figure 5: Antineutrino spectrum: prompt positron scattering[10]. ysis of chondrites, meteorites with the same composition of the Earth. After the first observation 
in 2010 and a revision in 2013, we present here the most recent results[10]. The main background component are the antineutrinos from power reactors, which we have carefully modelled from the fuel composition and up-time data provided by the power companies. Almost all other backgrounds are negligible, thanks to the tagging of the events given by the coincidence between the prompt positron scattering and the $\sim 250 \mu$ s-delayed $2.2 \mathrm{Mev}$ gamma from the $\mathrm{n}$ capture on $\mathrm{H}$. The total exposure is $(907 \pm 44)$ ton $\cdot y r$ where we select 77 golden coincidences. The prompt event energy spectrum can be seen in figure 5. An unbinned maximum likelihood fit returns $N_{\text {geo }}=23.7_{-5.7}^{+6.5}(\mathrm{stat})_{-0.6}^{+0.9}$ (syst) and $N_{\text {react }}=52.7_{-7.7}^{+8.5}(\mathrm{stat})_{-0.9}^{+0.7}$ (syst). The non observation of geoneutrinos is excluded at $5.9 \sigma$ level. The corresponding fluxes are $S_{\text {geo }}=(43.5 \pm 11.1) \mathrm{TNU}$ and $S_{\text {react }}=(96.6 \pm 15.9) \mathrm{TNU}$ where $1 \mathrm{TNU}=1 \mathrm{v} / 10^{32} \mathrm{protons} / \mathrm{yr}$. The flux of reactor antineutrinos compares well with the prediction of $(87 \pm 4) \mathrm{TNU}$, after neutrino oscillations are accounted for. Freeing the $\mathrm{Th} / \mathrm{U}$ ratio, we can resolve the two components, obtaining overall similar results and a ratio in good agreement with the chondritic ratio. We account for the local contribution of the crust $N_{\mathrm{LOC}}=(9.7 \pm 1.3) \mathrm{TNU}$ obtained by a a geological survey of the area around LNGS and the contribution of the Rest Of the Crust $S_{\mathrm{ROC}}=(13.7 \pm 2.5) \mathrm{TNU}$ as predicted by the planetary model. We can then exclude the absence of a Mantle contribution at 98\% C.L. and assign $S_{\text {mantle }}=20.9_{-10.3}^{+15.9}$ TNU. Our results are in good agreement with the Bulk Silicate Earth model predictions.

\section{Borexino Phase-II}

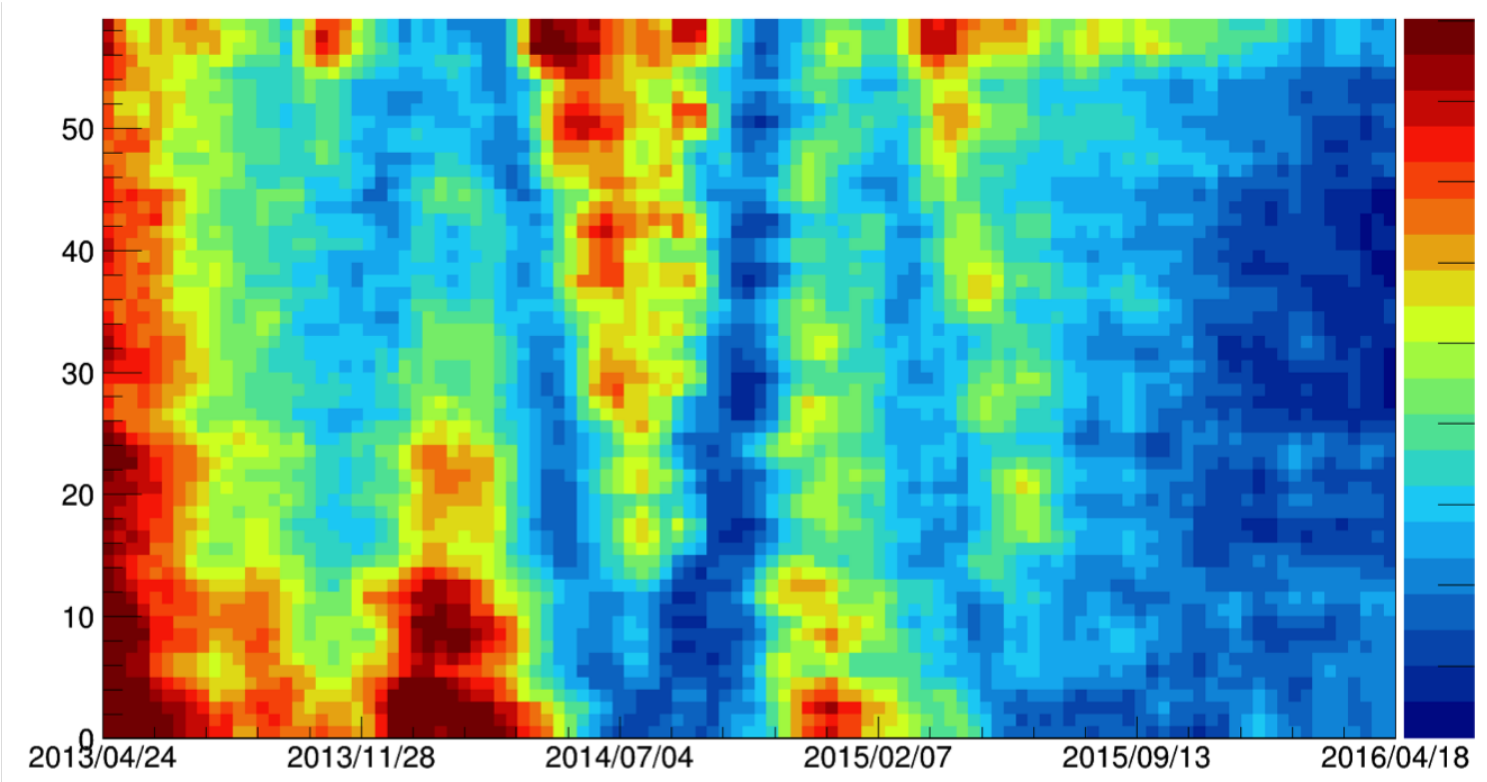

Figure 6: ${ }^{210}$ Po background rate. See text for description.

The Phase-II of the Borexino solar run will end with the deployment of the SOX antineutrino source, scheduled for the end of 2017. We will exploit the extended solar data to reduce the error on the ${ }^{7} \mathrm{Be}$ neutrino flux at $3 \%$ or better and to study its annual modulation, the ultimate proof we are actually observing neutrinos from the Sun. The ${ }^{8} \mathrm{~B}$ flux measurement will also be improved 
both in error (previously statistics dominated) and energy threshold. The most intriguing possibility we will pursue is however the improvement of the pep+CNO analysis with the twofold goal of (i) reducing the error on pep flux, obtaining an evidence at $>3 \sigma$, and (ii) lowering the upper detection limits on the $\mathrm{CNO}$ fluxes and eventually obtaining a first observation. In this respect we have improved the rejection capability of both the TFC and the PSA algorithms (Sect. 3) and we are now preparing a multivariate fit where the whole energy range is fitted simultaneously including the pp, the ${ }^{7} \mathrm{Be}$ and the pep+CNO regions. However it is mandatory to constrain the ${ }^{210} \mathrm{Bi}$ background due to its spectral quasi-degeneracy with the CNO neutrinos shape. Daughter ${ }^{210} \mathrm{Po}$ could be a good handle as the rate of this $\alpha$-decay can be easily measured with PSA. Unfortunately the equilibrium between ${ }^{210} \mathrm{Bi}$ and ${ }^{210} \mathrm{Po}$ is broken. Temperature fluctuations in the experimental Hall induce convective motions in the IV which bring particulate from the periphery into the FV. Consequently we observe a ${ }^{210} \mathrm{Po}$ rate which is the sum of a ${ }^{210} \mathrm{Bi}$-supported term and an unsupported term. Stopping convective motions, the unsupported ${ }^{210} \mathrm{Po}$ would decay with the mean life of $\sim 200 \mathrm{~d}$ and we could resolve the two terms. The supported term would then provide the independent constraint on

${ }^{210} \mathrm{Bi}$ background that is needed. During the second half of 2015 we have installed 65 new high sensitivity and stability probes that monitor the temperature of each detector's volume. We have also insulated the WT with a $20 \mathrm{~cm}$ layer of mineral wool and installed heaters on its top part to control the temperature at need. The goal of these efforts is to minimize external perturbations and establish a temperature gradient along the vertical axis of the detector. This would keep the scintillator stratified and prevent mixing dynamics. After a few month we can say that we are succeeding. Figure 6 shows the ${ }^{210}$ Po rate in arbitrary units in cubes of equal volumes indexed roughly from bottom to top of the IV and as function of date. Previously, summers induced high rates in the top of the detector, while the opposite was true for winters. After most of the insulation was in place in fall 2015 the ${ }^{210}$ Po rate started to decay naturally and uniformly across the detector.

\section{Conclusions}

Borexino has performed an almost full solar neutrino spectroscopy probing the Standard Solar Model and the MSW-LMA neutrino oscillation mechanism across the whole energy spectrum. The Borexino backgrounds are unprecedented low. The Phase-II of the solar run up the beginning of the SOX experiment[11] features six years of excellent quality data. We are working to obtain a more stringent limit on the CNO neutrino flux, hoping for an observation, along with more stringent measurements of ${ }^{7} \mathrm{Be},{ }^{8} \mathrm{~B}$, pep, and geo-neutrinos.

\section{References}

[1] W.C. Haxton et al., Ann. Rev. Astron. Astrophys. 51, 21 (2013).

[2] G. Bellini et al. (Borexino coll.), Nucl. Instrum. Methods A 609, 58 (2009).

[3] G. Bellini et al. (Borexino coll.), JINST 07, 10018 (2012).

[4] G. Bellini et al. (Borexino coll.), Phys. Rev. Lett. 107, 141302 (2011).

[5] G. Bellini et al. (Borexino coll.), Phys. Lett. B 707, 22 (2012).

[6] G. Bellini et al. (Borexino coll.), Phys. Rev. D 89, 112007 (2014)

[7] G. Bellini et al. (Borexino coll.), Phys. Rev. D 82, 033006 (2010).

[8] G. Bellini et al. (Borexino coll.), Phys. Rev. Lett. 108, 051302 (2012).

[9] G. Bellini et al. (Borexino coll.), Nature 512, 383-386 (2014).

[10] M. Agostini et al. (Borexino coll.), Phys. Rev. D 92, 031101(R) (2015).

[11] B. Neumair et al. (Borexino coll.), these proceedings. 